\title{
Heavy-Light Meson Semileptonic Decays with Staggered Light Quarks
}

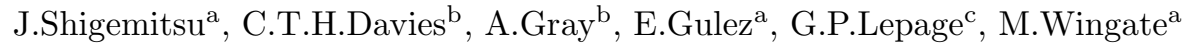 \\ a Physics Department, The Ohio State University, Columbus, OH 43210, USA. \\ ${ }^{\mathrm{b}}$ Department of Physics \& Astronomy, University of Glasgow, Glasgow, G12 8QQ, UK. \\ ${ }^{\mathrm{c}}$ Laboratory of Elementary Particle Physics, Cornell University, Ithaca, NY 14853, USA.
}

We report on exploratory studies of heavy-light meson semileptonic decays using AsqTad light quarks, NRQCD heavy quarks and Symanzik improved glue on coarse quenched lattices. Oscillatory contributions to three-point correlators coming from the staggered light quarks are found to be handled well by Bayesian fitting methods. B meson decays to both the Goldstone pion and to one of the point-split non-Goldstone pions are investigated. One-loop perturbative matching of NRQCD/AsqTad heavy-light currents is incorporated.

\section{Introduction}

A major systematic error in many studies of heavy-light meson decays has been the uncertainty due to chiral extrapolations in the light valence and light dynamical quark masses. These chiral extrapolation errors can be reduced significantly by working with light quark actions that have good chiral properties and this has motivated us to initiate a program to study heavy-light physics using improved staggered (or equivalently improved naive) light quarks coupled with NRQCD heavy quarks [1]. Our first results on $B$ and $D$ meson decay constants on the MILC dynamical configurations were presented by M.Wingate at this conference [2]. Here we discuss initial semileptonic decay results.

Our semileptonic studies are at a much more preliminary stage compared to the decay constant calculations. We report on quenched simulations with light quark mass around $m_{\text {strange }}$ and the heavy quark mass around the $b$-quark mass. We employ the Asqtad light quark action, the $O(1 / M)$ and $O\left(a^{2}\right)$ improved NRQCD heavy quark action and the tree-level Symanzik improved glue action. We work with $20012^{3} \times 32$ quenched configurations with $a^{-1} \approx 1.0 \mathrm{GeV}$.

\section{Fitting Oscillatory Three-point Corre- lators}

Two- and three-point correlators involving naive or staggered propagators have contributions that oscillate with time and this makes it more challenging to carry out fits and extract groundstate contributions. Experience gained recently with NRQCD/staggered heavy-light two-point correlators have demonstrated the usefulness of Bayesian fitting methods $[1,3]$. Hence, much of the effort in this exploratory study of semileptonic decays has focused on testing Bayesian fits to oscillatory three-point correlators. The basic correlator of interest is,

$$
\begin{aligned}
& C^{(3)}\left(\vec{p}_{\pi}, \vec{p}_{B}, t, T_{B}\right)= \\
& \sum_{\vec{z}} \sum_{\vec{y}}\left\langle\Phi_{\pi}(0) J^{\mu}(\vec{z}, t) \Phi_{B}^{\dagger}\left(\vec{y}, T_{B}\right)\right\rangle \\
& \quad \times e^{i \vec{p}_{B} \cdot \vec{y}} e^{i\left(\vec{p}_{\pi}-\vec{p}_{B}\right) \cdot \vec{z}}
\end{aligned}
$$

In the present simulations the $B$ meson decays at rest $\left(\vec{p}_{B}=0\right)$ and calculations have been performed with $T_{B}=12$ and $T_{B}=16$. The location of the current insertion $t$ was varied between 1 and $T_{B}$. The three-point correlator is fit to the form, 


$$
\begin{aligned}
& \sum_{k=0}^{C^{(3)}} \sum_{j=0}^{\left.\vec{p}_{\pi}, \vec{p}_{B}, t, T_{B}\right) \rightarrow} \\
& \quad \times A_{j, k} e^{-E_{\pi}^{(k)}(t-1)} e^{-E_{B}^{(j)}\left(T_{B}-t\right)}
\end{aligned}
$$

In this ansatz every second exponential comes with an oscillatory amplitude in both the pion and the $B$ channels, We were able to get good fits with $N_{\pi}=1$ or 2 and $N_{B}=3$ to 8 . Furthermore we found it useful to do simultaneous fits to $C^{(3)}$ and to the $B$ meson two-point correlator. Fits were done to all data between $t=2$ and $t=T_{B}-1$, i.e. to all but the first and the last timeslices, those timeslices on which the pion and the $B$ meson respectively were created. Figs. $1 \& 2$ give examples of fits to matrix elements of the temporal $\left(V_{0}\right)$ component of the heavy-light vector current. The plots show, $C^{(3)}\left(\vec{p}_{\pi}, t, T_{B}\right) e^{E_{\pi}^{(0)}(t-1)} e^{E_{B}^{(0)}\left(T_{B}-t\right)}$. By factoring out the groundstate contributions, the opposite parity oscillatory terms and other excited state contributions show up more clearly. Despite the presence of these excitations, the Bayesian fitting methods allow us to extract the groundstate amplitude of interest, $A_{00}$ in eq.(2).

The two figures are for the cases when the pion has momentum $(0,1,1)$ in units of $2 \pi /(a L)$. Good $\chi^{2} /$ dof were found with $N_{\pi}=1$ and $N_{B}=6$. The difference between the $T_{B}=12$ and $T_{B}=16$ results is typical for all matrix elements. With $T_{B}=12$, statistical errors are reduced and more accurate fits can be made. On the other hand, with $T_{B}=16$ a hint of a plateau can be observed (which will likely disappear with higher statistics and a more expanded vertical scale). Since with multi-exponential Bayesian fits the presence of a plateau is not required, one is better off with the more accurate $T_{B}=12$ data. Nevertheless, working with two different $T_{B}$ values provides a good consistency check, which we exploit in the next section.

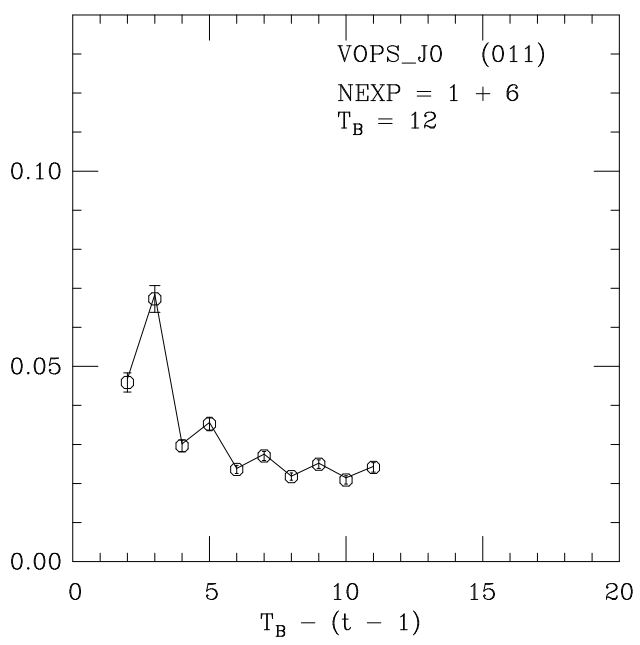

Figure 1. Fit results for the groundstate amplitude $A_{00}$ from the $\left\langle V_{0}\right\rangle$ three-point correlator with $T_{B}=12$. The pion momentum is $(0,1,1) 2 \pi /(a L) . N_{\exp }$ gives $N_{\pi}+N_{B}$.

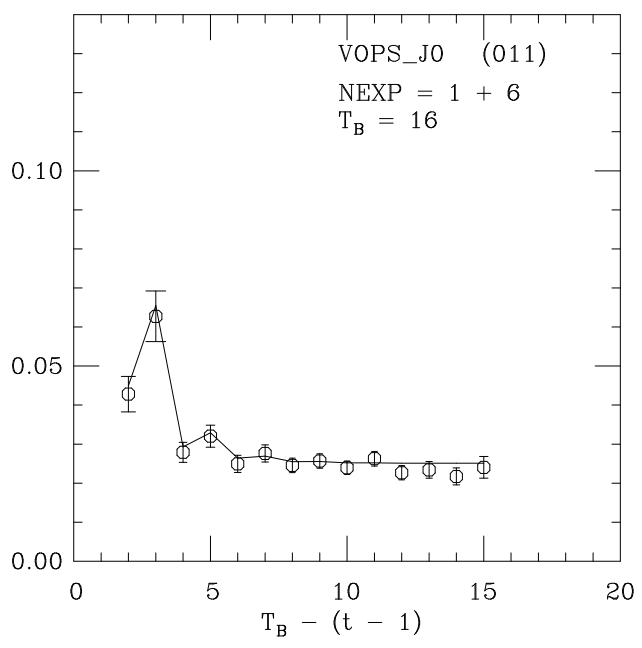

Figure 2. Same as Fig.1 but for $T_{B}=16$. 


\section{Results for Form Factors}

Given the amplitudes $A_{00}$ from fits to threepoint correlators one can determine various form factors. In these preliminary investigations we find it most convenient to work with the form factors $f_{\|}$and $f_{\perp}$ defined as,

$$
\begin{aligned}
f_{\|} & =\frac{A_{00}\left(V_{0}\right)}{\sqrt{\zeta_{\pi}^{(0)} \zeta_{B}^{(0)}}} \sqrt{2 E_{\pi}} Z_{V_{0}} \\
f_{\perp} & =\frac{A_{00}\left(V_{k}\right)}{\sqrt{\zeta_{\pi}^{(0)} \zeta_{B}^{(0)}} p_{\pi, k}} \sqrt{2 E_{\pi}} Z_{V_{k}}
\end{aligned}
$$

$\zeta_{\pi}^{(0)}$ and $\zeta_{B}^{(0)}$ denote the groundstate amplitudes of the pion and $B$ correlators respectively. The $Z_{V_{\mu}}$ are the matching coefficients for the heavylight currents. We use one-loop perturbative results for them.

In Fig. 3 we show results for $f_{\|}$and $f_{\perp}$. Our main results are the octagons and diamonds, which correspond to $T_{B}=12$ and $T_{B}=16$ results respectively, for decays into local Goldstone pions. One sees consistency between the two $T_{B}$ results, with, as expected, smaller statistical (and fitting) errors when $T_{B}=12$.

We have also considered $B$ decays to one of the point-split non-Goldstone pions, namely the onelink pion. The masses of the one-link and local pions differ as $M_{\pi}$ (one -link $) / M_{\pi}($ local $)=1.13$. The "bursts" in Fig.3 show form factors for the decay to the one-link pion. One sees that at low momentum agreement is reasonable with the local pion results. Things start to differ at higher pion momentum, however the data has started to deteriorate at those momenta and one also expects lattice artifacts to be worse there. Things should improve considerably as one goes to lattices finer than the $a^{-1} \approx 1 \mathrm{GeV}$ lattices of the present study. One can conclude from Fig. 3 that taste-breaking lattice artifact errors are smaller in form factors than in the corresponding pion masses, particularly at low pion momenta.

The above form factor results come from the lowest order (in $1 / M$ ) heavy-light currents. We have also analysed matrix elements of all the dimension 4 current corrections that come in at the next order (two additional currents for $V_{0}$ and

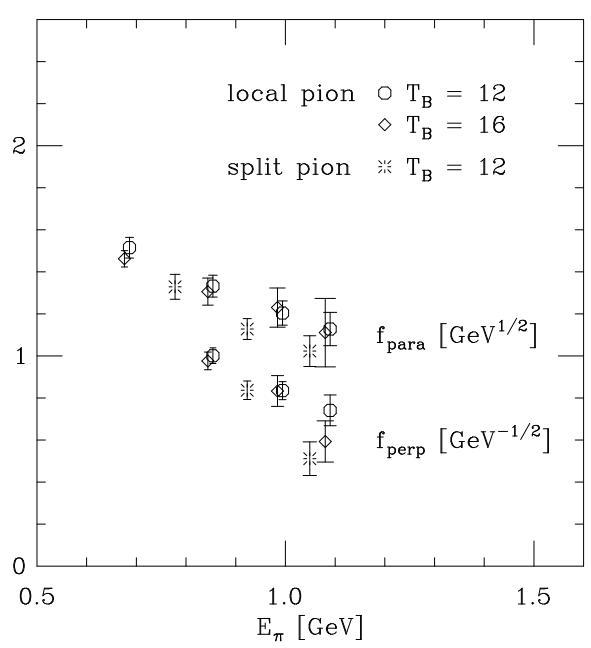

Figure 3. Semileptonic decay form factors $f_{\|}$and $f_{\perp}$ versus the pion energy $E_{\pi}$ in the $B$ rest frame.

four more for $V_{k}$ ). We find that their contributions are small for $B$ mesons giving at most a $\sim 2-3 \%$ correction.

\section{Summary}

Exploratory quenched studies of heavy-light meson semileptonic decays with Asqtad light quarks look promising. Simulations are underway to calculate form factors on the MILC dynamical configurations.

Acknowledgements : This work was supported by the DOE, PPARC and NSF. Simulations were carried out at the Ohio Supercomputer Center.

\section{REFERENCES}

1. M. Wingate et al.; Phys. Rev. D67, 054505 (2003).

2. M. Wingate et al.; these proceedings and in preparation.

3. G.P. Lepage et al.; Nucl. Phys. B(Proc. Suppl.)106, 12 (2002). 